\title{
Hypertensive Retinopathy and the Risk of Hemorrhagic Stroke
}

\author{
Ramani Thiagarajah, ${ }^{1}$ Regunath Kandasamy, ${ }^{2}$ Pulivendhan Sellamuthu ${ }^{1}$ \\ Department of Neurosurgery, Hospital Queen Elizabeth, Sabah, Malaysia \\ Department of Neurosciences, ${ }^{2}$ School of Medical Sciences, Universiti Sains Malaysia, Kelantan, Malaysia
}

Objective : Hemorrhagic stroke (HS) and hypertensive retinopathy are known end organ damage of the brain and eye respectively, with HS having deleterious consequence to the patients. This study is to correlate between hypertensive retinopathy and HS in hypertensive disease.

Methods : A control group of hypertensive patients only, and an investigated group of hypertensive HS patients. Fundoscopic examination to determine the grade of retinopathy was performed and then divided into low or high severity hypertensive retinopathy. Clinical and radiological parameter included are demography, vital signs, Glasgow coma scale (GCS) on admission, clot volume, site of clot, Intracerebral hemorrhage (ICH) score and Glasgow outcome scale (GOS). Data were correlated with the severity of hypertensive retinopathy.

Results : Fifty patient in the control group and 51 patients in the investigated group were recruited. In the hypertensive HS group, 21 had low severity retinopathy (no or mild retinopathy) accounting for $41.2 \%$ and 30 patients had high severity (moderate or severe retinopathy). In the hypertensive patients 49 had low severity and one had high severity ( $p$-value of 0.001$)$. In HS group low severity showed better GCS score of 9-15 on admission ( $p$-value of 0.003 ), clot volume less than $30 \mathrm{~mL}$ ( $p$-value 0.001 ), and also a better 30 days mortality rate by using the $\mathrm{ICH}$ score ( $p$-value 0.006 ), GOS score of 4 and 5 the low severity retinopathy fair better than the high severity retinopathy ( $p$-value of 0.001 ), and the relative risk to develop HS in low severity and high severity retinopathy was 0.42 and 29.4 , respectively.

Conclusion : Hypertensive retinopathy screening could be used as an indicator in hypertensive patient, to evaluate the risk of developing hypertensive $\mathrm{HS}$ in the future.

Key Words : Hypertensive retinopathy · Hemorrhagic stroke.

\section{INTRODUCTION}

Hemorrhagic stroke (HS) secondary to hypertension accounts for up to $15 \%$ of cases in all types stroke and carries a mortality rate about 38\% within the first year. In the world population there is higher percentage of HS in Southeast Asia. Studies in contrast to the Caucasian population in Australia, with the Asian population showing a five times higher incidence of developing $\mathrm{HS}^{6)}$. Malaysia being a multicultural society also shows a disparity among the ethnic and major race

- Received : September 28, 2020 •Revised : December 2, 2020 •Accepted : December 14, 2020

- Address for reprints : Ramani Thiagarajah

Department of Neurosurgery, Hospital Queen Elizabeth, 13a Jalan Penampang, Kota Kinabalu, Sabah 88200, Malaysia

Tel : +6016-8105890, Fax : +6088-211999, E-mail : ramani.@@gmail.com, ORCID : https://orcid.org/0000-0002-9484-3388

This is an Open Access article distributed under the terms of the Creative Commons Attribution Non-Commercial License (http://creativecommons.org/licenses/by-nc/4.0) which permits unrestricted non-commercial use, distribution, and reproduction in any medium, provided the original work is properly cited. 
group, with the indigenous race from the state of Sabah and Sarawak accounting for a prevalence of $36.4 \%$, followed by Malays at 34\%, Chinese at 32.3\% and lastly Indian 30.6\%.

Retinopathy changes in the eye due to hypertension is a chronic process, resulting pathophysiological changes that occur in the retinal, choroidal and optic nerve circulation are all in response to elevated blood pressure (BP) resulting in retinopathy changes in these structures ${ }^{12)}$. As for the pathogenesis of hypertensive HS, caused tiny lipohyalinotic aneurysms formation called Charcot-Bouchard aneurysm along the lenticulostriate arteriole supplying the basal ganglia region which when rupture, resulting in intra parenchymal hemorrhage ${ }^{3)}$. The intracranial and optic vasculature system, share similar anatomical, physiological and embryological characteristic. Possessing a blood-retinal barrier which is identical to brainblood barrier, and the retina being an extension of the diencephalon, thus we could hypothesize the changes in the cerebral vessel could be similar to the retinal vessel hence a fundoscopic examination of the retinal vessel may provide an opportunity to understand the intracranial vessel changes and the development of HS secondary to hypertension ${ }^{1,8,11)}$.

The most recent study conducted on the Atherosclerotic Risk in Communities (ARIC) subjects by Ong et al.' in 2013, of the 2907 subject, with no retinopathy changes, mild retinopathy changes and moderate or malignant retinopathy changes, of which 146 developed cerebral infarctions and 15 were HSs. The incidence of stroke events for the whole population was 0.436 per 100 person-years (95\% confidence interval [CI], 0.42-0.45), 0.322 per 100 person-years (95\% CI, $0.305-0.339)$ for the group with no retinopathy, and 0.493 per 100 person-years (95\% CI, 0.466-0.519) and 1.073 per 100 person-years (95\% CI, 0.899-1.246) for the group with mild and moderate hypertensive retinopathy, respectively ${ }^{7)}$. Many studies conducted were mostly on cerebral infarction, and the main pathogenesis is atherosclerosis. There have not been any studies correlating hypertensive retinopathy with HS secondary to hypertension, therefore it can be postulated that there is an association of the pathogenesis of HS due to hypertension and eye signs. The regional neurosurgery center in Kota Kinabalu, covers a great deal of the state. This includes the capital state, Kota Kinabalu and around 25 districts primary centres, where our hospital is the tertiary referral centre. This covers approximately a population of 1.8 million people, giving a good demographic study of the population of Borneo, which consist of indigenous native (Kadazan, Dusun, Murut, and Bajau), Malays, Chinese, and other minorities. The study aims to determine the prevalence of hypertensive retinopathy changes and hypertensive HS based, on the severity of hypertensive retinopathy, Glasgow coma scale (GCS), Intracerebral hemorrhage (ICH) score to access the 30 days mortality rate, and clot volume. To date no studies similar were conducted among HS, this will be the first study conducted, and will help determine the association of hypertensive retinopathy changes among HS patients.

\section{MATERIALS AND METHODS}

The study was conducted between May 2016 and May 2018. The study was approved by the Malaysian Medical Research and Ethics Committee (NMRR ID : NMRR-16-2691-32571).

The HS group inclusion criteria were all spontaneous intracranial hemorrhage due to primary hypertension referred to our center, and between the age of ranging from 35-70 years old. The exclusion criteria patient's with intracranial hemorrhage due to trauma, coagulopathy, vascular abnormality including subarachnoid hemorrhage, infective cause, tumoral bleed and cerebral amyloid angiopathy or with known secondary cause of hypertension from endocrine, renal, adrenal or thyroid disease. Patient's with spontaneous intracerebral hemorrhage but are not known to be hypertensive prior to admission and patient with co-morbid of diabetes mellitus, ischemic heart disease, a history of cerebral infarction, patient on anticoagulants therapy, patient on antiplatelet therapy, hemorrhagic transformation of ischemic stroke or sustain any eye or cranial injuries and diseases were also excluded.

For the control/non-HS group which consist of hypertensive patients, on follow up in the community clinic. Inclusion criteria was age ranging from 35 till 70 years old and on treatment for primary hypertension. Exclusion criteria, patient with all types of HS, cerebral infraction, on any anticoagulant or antiplatelet medication, brain tumor and any form of cerebral vascular malformation, any secondary causes for hypertension, patients with co morbid of diabetes mellitus, ischemic heart disease and sustain any eye or brain injuries and diseases.

Prospective nonrandomized patient once diagnosed with hypertensive hemorrhage based on computed tomography 
(CT) scan on admission fulfilling the inclusion and exclusion criteria were recruited for the HS group. Patients underwent treatment in accordance with standard protocol for hypertensive HS. Clinical and radiological parameter were collected in a collection data entry form, the parameter includes patient age, sex, race, BP, GCS on admission, clot volume size, origin of hemorrhage and the presence of intraventricular hemorrhage, treatment or intervention performed to the patient and Glasgow outcome scale (GOS). ICH score is a prognostication based on 30 day mortality rate, it was done by allocating points based on GCS score of 3-4, 5-12, and 13-15, with a point of 2, 1, and 0 , respectively, the clot volume of more or equal of $30 \mathrm{~cm}^{3}$ having 1 points and 0 if it is less than $30 \mathrm{~cm}^{3}$, the presence of intraventricular hemorrhage, 1 point if presence, 0 point in absence, infratentorial origin of clot gets 1 point, with 0 points for other site of origin, and lastly age 80 years old and beyond having 1 point and 0 point for those less than 80 years, the cumulative points of 0 till 6 will be calculated for the patients. Retinal examination picture was taken with a digital fundoscopic camera apparatus using PanOptic Ophthalmoscope (Welch Allyn Inc., Skaneateles Falls, NY, USA) that was recorded and stored as photo or image, within the first 24-48 hours, after the patients were clinically stabilized. The severity was graded on both the fundi, based on Wong and Mitchell classification of hypertensive retinopathy $^{7)}$, Table 1 . The retinal picture was accessed by two independent ophthalmologist that were blinded to the patient clinical condition.

Control/non-HS group were prospective non-randomized patient who fulfilled the inclusion and exclusion criteria were recruited during their follow up hypertension visit in the community clinic. The clinical parameter was taken, like BP, during the visit to the clinic, with the HS patients, and retinal examination picture of the fundi were carried out and ac- cessed by two independent ophthalmologist that were blinded to the patient clinical condition based on Wong and Mitchell classification of hypertensive retinopathy ${ }^{7}$.

\section{Statistical analysis}

The sample size was calculated using PS sample size software (Stephens J, Plummer WD, Blume JD, Dupont WD, Vanderbilt University School of Medicine, Nashville, TN, USA), with a power of $0.8, a$ of $0.05, \mathrm{P}_{0}$ of 0.14 , rate for the control group and $\mathrm{P}_{1}$ of 0.4 , rate for investigate group based of the ARIC study, a sample size of 45 patients in each group was determined, with a dropout rate of $10 \%$, the total sample size for each group is 50 patients. Numerical variables will be presented in mean and standard deviation whereas categorical variables will be presented in frequency and percentage. Pearson chi-square test was used to determine the association between hypertensive retinopathy and HS based on the parameters and the comparison of the BP between the control and HS group.

\section{RESULTS}

One hundred and one patients were recruited in the study, 51 patients with hypertension and HS, as the investigated arm and 50 patients with hypertensive disorder but no event of HS taken as a control group that satisfy the inclusion criteria of the study. The age group include from 35 years old till 70 years old, Mean age of the patients 54.0 (standard deviation [SD], 9.2). The demographic distribution, $58.4 \%$ subjects were male and $41.6 \%$ were female, with majority of patient Chinese $(n=31)$, followed by the ethnic group of Sabah, Dusun $(n=20)$, and Kadazan $(n=19)$, other ethnic group of Sabah accounting for 28 patients and Malay patients being the least with three

Table 1. Mitchell and Wong classification for hypertensive retinopathy ${ }^{7)}$

\begin{tabular}{ll}
\hline Grade & Do detectable retinal sign \\
\hline $\begin{array}{l}\text { No retinopathy } \\
\text { Mild retinopathy }\end{array}$ & $\begin{array}{c}\text { One or more of the arteriolar signs : generalized arteriolar narrowing, focal arteriolar narrowing, arteriovenous nicking, } \\
\text { arteriolar wall opacity (silver wiring) }\end{array}$ \\
Moderate retinopathy & $\begin{array}{c}\text { One or more of the following retinal signs : hemorrhage (dot, blotter flamed shaped), microaneursym, cotton wool spot, } \\
\text { hard exudates }\end{array}$ \\
Malignant retinopathy & Moderate retinopathy plus optic disc swelling \\
\hline
\end{tabular}


patients, as in Table 2.

The BP for the entire population, ranges from 122-268 $\mathrm{mmHg}$ for systolic, with mean 166.36 (SD, 31.734), and for diastolic 64-148 mmHg with mean 94.53 (SD, 16.05). As for the HS group, the systolic BP, mean 187.95 (SD, 29.66) and diastolic BP mean 99.24 (SD, 20.68) that was taken during the acute stage, which was higher than the non-HS (control group) had systolic BP mean 144.34 (SD, 13.34) and diastolic BP mean 89.74 (SD, 6.55) this was statistically insignificant with a $p$-value of 0.012 for the systolic BP and $p$-value of 0.010 for diastolic BP using the Pearson chi-square test comparing the HS and the non-HS group. The data collected in the HS group, with GCS of 3-8 accounting for 17 (33.3\%), 19 (37.3\%) having GCS 9-12, and GCS of 13-15 having 15 (29.4\%). Two main grouping was done for GCS, a score of 3-8 having 17 patients considered poor conscious level on admission and GCS of 9-15 with 34 patients, considered good conscious level on admission. The distribution for site of clot are putamen 30 (58.8\%), thalamus 13 (25.5\%), followed by cerebellar, brain

Table 2. Patients demographic distribution in study

\begin{tabular}{lc}
\hline & Value \\
\hline Age (years) & $54.0 \pm 9.2$ \\
Sex & $59(58.4)$ \\
Male & $42(41.6)$ \\
Female & \\
Race & $3(3.0)$ \\
Malay & $31(30.7)$ \\
Chinese & $20(19.8)$ \\
Dusun & $19(18.8)$ \\
Kadazan & $28(27.7)$ \\
Others & \\
Group & $51(50.5)$ \\
Investigated group (HS) & $50(49.5)$ \\
Control group (non HS)
\end{tabular}

Frequency of HS

$\begin{array}{lc}\text { Putamen } & 30(58.8) \\ \text { Thalamus } & 13(25.5) \\ \text { Cerebellar } & 4(7.8) \\ \text { Brain stem } & 3(5.9) \\ \text { Lobar } & 1(2.0)\end{array}$

Values are presented as mean \pm standard deviation or number (\%). HS : hemorrhagic stroke stem and lobar, four, three, and one patients, respectively. The results for clot volume less $30 \mathrm{~mL}$ by CT, accounts for 30 of the patients (58.8\%) and clot volume more then $30 \mathrm{~mL}$ having 21 patients (41.2\%). ICH score was calculated on admission for all the HS, ICH score from 0-2 had 42 patients, and ICH score of 3-6 had nine patients. The GOS score were taken with fa-

Table 3. Data of HS group and non HS group

\begin{tabular}{|c|c|c|}
\hline Criteria & HS group & Non HS group \\
\hline \multicolumn{3}{|l|}{ Blood pressure } \\
\hline Systolic & $187.95 \pm 29.66$ & $144.34 \pm 13.34$ \\
\hline Diastolic & $99.24 \pm 20.68$ & $89.74 \pm 6.55$ \\
\hline \multicolumn{3}{|l|}{ GCS } \\
\hline $3-8$ & $17(33.3)$ & \\
\hline $9-12$ & $19(37.3)$ & \\
\hline $13-15$ & $15(29.4)$ & \\
\hline \multicolumn{3}{|l|}{ GCS group } \\
\hline 3-8, poor on admission & $17(33.3)$ & \\
\hline 9-15, good on admission & $34(66.7)$ & \\
\hline \multicolumn{3}{|l|}{ Clot volume } \\
\hline Less than 30 mL & $30(58.8)$ & \\
\hline More than $30 \mathrm{~mL}$ & $21(41.2)$ & \\
\hline \multicolumn{3}{|l|}{$\mathrm{ICH}$ score } \\
\hline 0 & $7(13.7)$ & \\
\hline 1 & $13(25.5)$ & \\
\hline 2 & $22(43.1)$ & \\
\hline 3 & $5(9.8)$ & \\
\hline 4 & $4(7.8)$ & \\
\hline 5 & $0(0.0)$ & \\
\hline 6 & $0(0.0)$ & \\
\hline \multicolumn{3}{|l|}{$\mathrm{ICH}$ group } \\
\hline $\mathrm{ICH} 0-2$ & $42(82.4)$ & \\
\hline ICH 3-6 & $9(17.6)$ & \\
\hline \multicolumn{3}{|l|}{ Intervention } \\
\hline Conservative & $21(41.2)$ & \\
\hline EVD & $9(17.6)$ & \\
\hline Surgery & $21(41.2)$ & \\
\hline \multicolumn{3}{|l|}{ GOS } \\
\hline Favourable outcome, GOS 4-5 & $24(47.1)$ & \\
\hline Unfavourable outcome, GOS 1-3 & $27(52.9)$ & \\
\hline
\end{tabular}

Values are presented as mean \pm standard deviation or number (\%). HS : hemorrhagic stroke, GCS : Glasgow coma scale, ICH : Intracerebral hemorrhage, EVD : external ventricular drainage, GOS : Glasgow outcome scale 
vorable outcome (GOS score, 4-5) in 24 patients (47.1\%), and unfavourable outcome in 27 patients (52.9\%). Twenty-one patients in the study were treated conservatively and another 21 patients were treated surgically, another nine patients had only cerebrospinal fluid diversion, all these are depicted in Table 3.

The fundoscopic examination was reviewed and classified according to Wong and Mitchell classification of hypertensive retinopathy ${ }^{7)}$ as shown in Table 1. We then grouped normal and mild retinopathy as low severity retinopathy and moderate and severe retinopathy as high severity retinopathy. The reason was in HS group, there were mainly two types of retinopathy grade mild and moderate, with no malignant grade were detected. As for the non-HS group, 49 patients had either no or mild retinopathy changes and only one patient with moderate retinopathy, therefore we define low severity retinopathy by grouping the no and mild retinopathy changes. Moderate retinopathy changes were found in 30 HS group and one non-HS group, we had no malignant retinopathy changes in our study, therefore we define the moderate and malignant as high severity retinopathy. In the non-HS group 49 patients has low severity and only one presented with high severity. Whereas in the HS group, 21 had low severity accounting for $41.2 \%$ and 30 patients had high severity as depicted in Table 4 , this comparison was significant with a $p$-value of less than 0.001. Fundoscopic examination for both the eyes were recorded and interpreted by two independent accessors, and an inter-rater bias analysis was done using Cohen Kappa test with a value of $\kappa=0.94$ for the right eye, and $\kappa=0.985$ for the left eye,

Table 4. Distribution of retinopathy in hypertensive patient with and without hemorrhagic stroke low-normal and mild retinopathy, highmoderate and severe retinopathy

\begin{tabular}{lccc}
\hline Retinopathy severity & HS & Non HS & p-value \\
\hline Low & $21(41.2)$ & $49(98)$ & \\
High & $30(58.8)$ & $1(2.0)$ & $<0.001$ \\
\hline
\end{tabular}

Values are presented as number (\%). HS : hemorrhagic stroke

Table 5. Hypertensive retinopathy and GCS

\begin{tabular}{|c|c|c|c|}
\hline \multirow{2}{*}{ Retinopathy severity } & \multicolumn{2}{|c|}{ GCS } & \multirow{2}{*}{$p$-value } \\
\hline & $3-8$ & 9-15 & \\
\hline Low & 2 & 19 & \\
\hline High & 15 & 15 & 0.003 \\
\hline
\end{tabular}

GCS : Glasgow coma scale giving almost perfect agreement between accessors.

We analyse the association of hypertensive retinopathy severity in the HS group with GCS on admission, clot volume, ICH group and GOS score using Pearson chi square test. When analysed with GCS on admission, low severity had a total of 21 patients of which 19 patients had GCS between 9 and 15, and only two patients had GCS 3-8. For high severity, a total of 30 patients with 15 patients in each GCS of 3-8 and 9-15, that was statistically significant with a $p$-value of 0.003 , as depicted in Table 5 .

Low severity also showed a clot volume less then $30 \mathrm{~mL}$ in 19 patients with only two patients having a clot volume more than $30 \mathrm{~mL}$. In high severity, 11 patients had clot volume less than $30 \mathrm{~mL}$, and 19 patients had clot volume more then 30 $\mathrm{mL}$, the chi square test reveal a $p$-value of 0.001 , also statically significant, as depicted in Table 6. ICH score that was calculated on admission was group into ICH 0-2 that had lower 30 days-mortality rate up to $26 \%$ and having a better prognosis, ICH 3-6 that carries a high 30 days-mortality rate above $72 \%$, hence a poorer prognosis, when analysed low severity only had ICH score between 0-2 accounting for 21 patients, as for high

Table 6. Hypertensive retinopathy and clot volume

\begin{tabular}{|c|c|c|c|}
\hline \multirow{2}{*}{ Retinopathy } & \multicolumn{2}{|c|}{ Clot volume (mL) } & \multirow{2}{*}{$p$-value } \\
\hline & $<30$ & $>30$ & \\
\hline Low & 19 & 2 & \\
\hline High & 11 & 19 & 0.001 \\
\hline
\end{tabular}

Table 7. Hypertensive retinopathy severity and ICH score group

\begin{tabular}{lccc}
\hline & \multicolumn{3}{c}{ ICH score group } \\
\cline { 2 - 4 } Retinopathy severity & ICH score & ICH score & p-value \\
& $\mathbf{0 - 2}$ & $\mathbf{3 - 6}$ & \\
\hline Low & 21 & 0 & \\
High & 21 & 9 & 0.006 \\
\hline
\end{tabular}

$\mathrm{ICH}$ : intracerebral hemorrhage

Table 8. Hypertensive retinopathy severity and GOS

\begin{tabular}{lccc}
\hline \multirow{2}{*}{ Retinopathy severity } & \multicolumn{3}{c}{ GOS } \\
\cline { 2 - 4 } & GOS 4-5 & GOS 1-3 & p-value \\
\hline Low & 17 & 4 & \\
High & 7 & 23 & 0.001 \\
\hline
\end{tabular}

GOS : Glasgow outcome scale 
severity 21 had ICH score $0-2$, while another nine patients had ICH score of 3-6, the $p$-value calculated was 0.006 , as depicted in Table 7. Lastly, we compared with the GOS score of the patient, we found favourable outcome of GOS score 4-5, low severity of retinopathy accounted for 17 patients, with only four having unfavourable outcome (GOS score, 1-3). As for high severity, 23 patients had unfavourable outcome with seven having favourable outcome, this was statistically significant having a $p$-value of 0.001 , as depicted in Table 8 . The relative risk (RR) for patient with high severity having HS is 29.4, while for low severity is 0.42 . But the low severity retinopathy required recalculation, within the non-HS group 42 (84\%) had no retinopathy changes and seven (14\%) had mild retinopathy changes, while the HS group 21 (41.2\%) had mild retinopathy changes and none had no retinopathy, so the RR for no retinopathy changes is 0.49 and 2.94 if mild retinopathy changes is detected. For the high severity, all the patient in HS and non-HS group had only moderate retinopathy changes, none were grade as malignant retinopathy.

\section{DISCUSSION}

There has been studies done to correlate hypertension retinal study with renal disease, even prospective one, but were not conclusive, as seen in the ARIC study, Beaver Dam Eye Study and Cardiovascular health study ${ }^{2,9,13)}$. A similar study was also conducted for retinopathy association with cardiovascular disease (CVD), to predict the mortality, the Blue Mountain Eye Study, showed association of CVD with mortality in patients with retinopathy ${ }^{4}$. Ibaraki Prefectural Study, describe patients with mild retinopathy changes regardless of hypertensive status, were at risk of CVD mortality, giving a prognostic value of retinopathy changes in $\mathrm{CVD}^{10)}$. Based on the study Hypertensive Retinopathy and Risk of Stroke ${ }^{7)}$, it was established that the moderate retinopathy had higher risk of developing stroke, but it was mainly cerebral infarct seen, with the hazard ratio (HR) 1.39 for mild retinopathy, and HR of 2.20 for moderate retinopathy ${ }^{7)}$. Unfortunately there is a lacking of studies that compare hypertensive retinopathy with its association with HS secondary to hypertension, as the number of affected patients are relatively lower, therefore, we decided to take a approach to capture the data of admitting patient within our neurosurgical centre with HS, and to detect severity of retinopathy, for the possibility to shed some light on the association between hypertension retinopathy and HS. But many studies showed retinopathy changes are associated with a varying type of disease. Patient with renal disease, cardiac disease and diabetic mellitus or elevated blood sugar level on admission were excluded as it will affect retinopathy changes and are confounding factors in the study. This were the major factors that required to be excluded in the study because the pathogenesis of the retinopathy were artherosclerosis and diabetic vasculopathy.

The BP for the HS group was higher on admission to hospital within the first 24 hours as compared to the non-HS group, the BP was taken during the first day of presentation, with mean systolic BP, $187.95 \mathrm{mmHg}$ and, mean diastolic BP 99.24 mmHg. The non-HS group had a mean systolic of 144.34 $\mathrm{mmHg}$ and mean diastolic of $89.74 \mathrm{mmHg}$, that was lower but not statistically significant. In the HS group, elevated BP is due to existence of the underlying primary hypertension, along with other factors. In patient with chronic hypertension, the relationship of cerebral blood flow and the cerebral perfusion pressure (CPP) curve is shifted to the right and resulting in a higher CPP, in turn results to a higher BP or mean arterial pressure, this is a physiological response for autoregulation of the brain in normal condition. In HS patients due to the longstanding hypertension the brain compliance is poor, resulting in auto dysregulation of the brain, that may further worsen the cerebral oedema in addition to the mass effect caused by intracerebral hematoma, the intracranial pressure exerted to the brain increases causing the BP to rise, to maintain the CPP of the brain thus forming a vicious cycle resulting elevation of the BP. This scenario is common in clinical setting, we encounter HS patient that are resistant to multiple antihypertensive medication during the course of treatment in the hospital. The control group were already on long term medication, regular follow up and were relatively healthy individuals, therefore control of their BP was better, despite some having retinopathy changes it is possible there are in the early stages of hypertensive disease. Over the course of treatment for HS, most patient BP did improve over time, but this was due to intervention to relieve the mass effect and medication, but the response did vary from one patient to another, in term of the duration to improve.

Patient within the HS group definitely suffer from some form of retinopathy be it mild or moderate, this reveals that 
the retinal vasculature do share some similarities with the cerebral vasculature, and due to elevation of BP and may result damage in the cerebral vasculature and results into HS as a consequence. Postulating that the HS may have an association with the formation of hemorrhages within the fundus due to ruptured micro-aneurysm, to some extent may confirm the formation of cerebral vasculature Charcot-Bouchard aneurysm that is responsible to intracranial hematoma caused by its rupture. The retinopathy changes that correspond to mild retinopathy, may represent an early formation of vasculature disease. The changes that were observe in the retinopathy, makes it a valuable indicator to ascertain the risk of HS in patients. Retinopathy screening for hypertensive patients performed routinely may serve a warning and warrants closer follow up or preventive measure may be taken for patient due to the prevalence of developing HS.

In the non HS group 42 patients had no retinopathy changes and only seven presented with mild retinopathy changes, whereas in the HS group there were 21 patients had mild retinopathy changes and 30 patients had moderate retinopathy changes. In the ARIC study in 2013 ${ }^{7}$, the incidence of stroke for the normal and mild retinopathy was 0.322 and 0.493 , respectively, that was similar with the overall incidence of stroke in the ARIC study that was $0.436^{7)}$, based on this reference in our study we define the no and mild retinopathy changes as low severity retinopathy. The moderate and malignant retinopathy are more likely due to chronic and advance end organ damage of the eye, similarly by using the ARIC study as reference the incidence of stroke was 1.073 in the moderate retinopathy group ${ }^{7)}$, which was higher than the overall incidence of stroke in the study and with only one patient having malignant retinopathy, and was group together with the moderate retinopathy group, therefore based higher incidence we define the moderate and malignant retinopathy grade to high severity retinopathy.

From this definition, low severity retinopathy reveals that in HS group suffered by the patients reveal a relatively lower clot volume, which is less then $30 \mathrm{~mL}$, this would explain the better GCS on admission, and ICH score due to less mass effect exerted on the brain parenchyma. Low severity retinopathy is more likely to be treated medically, hence reducing chance of developing post-operative complication, this translates to a better favourable outcome is more likely in the low severity group. The RR for developing of HS in the low severity reti- nopathy was 0.42 , but this may not be representative of this group, during the duration of the study, the non-HS group, had a large population that had no retinopathy changes and only seven had mild retinopathy changes. When correcting for this discrepancy, in the no retinopathy changes the RR for patient with no retinopathy changes was 0.49 while the mild retinopathy changes has a $\mathrm{RR}$ of 2.94 . This was more representative of the risk of HS, patient with no retinopathy changes with underlying hypertension, had a lower chance to develop HS and we term as low risk group, we suggest that regular yearly or 2 yearly retinopathy screening for patient, followed by with lifestyle modification, effective of hypertension treatment, and regular BP monitoring would suffice, in this stage importance should be placed in preventive measures to avoid end organ damage of the not just the brain, but even the cardiac and renal system. In the mild retinopathy grade, we observe an increase up to 3 folds the risk of HS, making this group a moderate risk group, patients will need regular follow up for hypertension, and optimization of medication quickly, the presence of retinopathy changes indicates that other organ may also suffer similar insult and it should be address. Retinopathy screening probably will need to be done at least 6 monthly or yearly. In this group, treatment and to prolong the development of HS should be the main strategy.

For high severity retinopathy, the clot volume tends to be larger and presenting GCS to hospital is poor, the ICH score of these patients is higher hence a poorer prognosis. The high severity retinopathy group could explain the chronic damage to the retinal and cerebral vessel wall, as the arteriole wall would have been severe damage and possible multiple area of micro aneurysm were formed, causing a significant larger clot formation when it ruptured, mass effect in the brain, and poor GCS on admission. Most likely subjecting the patient for surgical intervention based in the prognosis. The outcome of the patients tends to be poor this can be seen in the ICH score. Patients with HS are also prone to many infections, and complication due to being bed bound, which compounds the poor outcome of the patients. The RR of developing HS in the high severity retinopathy is almost 30 times, making it a high-risk group, therefore strategy for treatment in this group should be in anticipation of complication. This group of patient may benefit from tertiary hospital care and follow up, aggressive control of BP, patient or family members be made aware of the high probability of HS, retinopathy screening here, may not 
need to be frequent, but more importantly should be done to manage the complication that developed due to secondary damage sustain by the eye along with the brain and that of other organ like the heart and renal that may be compromised already. By defining the risk group for the patient, we are better able to treat the patient more objectively and may be able to detect patient in the early stage of hypertension complication so the preventive measure may be taken to avoid HS. With the RR establish, hypertensive retinopathy severity may be used as a predictor for HS for patients in the future.

\section{Limitation of the study}

Firstly, the study was not randomised, ICH score of 4 in our study were, treated conservatively because during initial admission to our centre the neurological status was poor. Because of this we could not find any correlation with the need for surgery and was expected to have bad outcome. The ICH score 2 was the largest group and during the study many were treated surgically, due to mass effect, a further analysis should be done within this group to ascertain the likely hood of this group to require surgical treatment or not. Within the study malignant retinopathy was not detected, a few reasons could be accounted for, the fundoscopic will require patient to have some degree of cooperation to be perform on, therefore it is possible that such patient would have been missed. Also case of severe retinopathy, is rare, reported as $1 / 2907$ in the one study ${ }^{7)}$, and $0.30 \%$ in another study therefore in a larger population it could have been detected ${ }^{5)}$. There are certain confounding factor that could not be correct adequately, like sex, race. Other factor that we did not take into account was cigarette smoking, body mass index (BMI) and alcohol consumption.

\section{Future recommendation}

A larger population should be studied, as it will provide a good local data, as the population of Sabah is multi-racial, will help correct the cofounding factors based on race, along with sex and age. Other factor should be include in the study are BMI, alcohol consumption and cigarette smoking. Randomization of patients would also be possible in larger population study, that may provide a better RR for HS. This could be possible if the patient were followed up over a long period of time around 10-15 years in the study. In term of neurosurgery, if retinopathy changes can be associated with the need for surgical intervention, hence screening of retinopathy, may reveals the risk group of patients that predispose for surgery, and could be monitored in tertiary centre with the present of a neurosurgery consult. This screening is also a preventive measure for patients, and a treatment and management of hypertension disease and its complications.

\section{CONCLUSION}

The severity of retinopathy changes detected in the HS patient provides us a glimpse of the outcome. High severity retinopathy translates to poor prognostication and unfavourble outcome as compared to low severity retinopathy. This compounded by risk post-operative complication, requiring extensive stroke rehabilitation and social as well as financial burden. Hypertensive retinopathy screening could be used as an indicator in hypertensive patient, to evaluate the risk of developing hypertensive HS in the future. The screening, instead will facilitate proper course of action based on the risk group, from lifestyle modification, medication, follow up and tertiary consultation for high risk group.

\section{CONFLICTS OF INTEREST}

No potential conflict of interest relevant to this article was reported.

\section{INFORMED CONSENT}

This type of study does not require informed consent.

\section{AUTHOR CONTRIBUTIONS}

\author{
Conceptualization : RT \\ Data curation : RT \\ Formal analysis : RT, RK \\ Funding acquisition : RT \\ Methodology : RT, RK \\ Project administration : RT, RK \\ Writing - original draft : RT, RK, PS \\ Writing - review \& editing : RT, RK, PS
}




\section{ORCID}

Ramani Thiagarajah https://orcid.org/0000-0002-9484-3388

Regunath Kandasamy https://orcid.org/0000-0002-8676-2633

Pulivendhan Sellamuthu https://orcid.org/0000-0002-2362-8162

\section{- Acknowledgements}

I would like to express my sincere gratitude and appreciation to all those who have directly or indirectly aid me in completing this thesis. First and foremost, I would like to express my utmost gratitude and appreciation to my dissertation supervisors Datuk Mr. (Dr.) Pulivendhan Sellamuthu (Head of Department of Neurosurgery, Queen Elizabeth Hospital, Kota Kinabalu, Sabah) and Mr. (Dr.) Regunath Kandasamy (Consultant Neurosurgeon, Universiti Sains Malaysia, Kubang Kerian, Kelantan). Their motivation, patience, continuous guidance, and encouragement has brought me towards completing this work.

I also would like to take this opportunity to thank the founder of the Neurosurgical Residency training Programme in Universiti Sains Malaysia, Professor Dato' Mr. (Dr.) Jafri Malin Abdullah and Head of Neurosciences Department, Universiti Sains Malaysia, Professor Mr. (Dr.) Zamzuri Idris. Their advices, guidance, and encouragement are invaluable. Special thanks to Miss. Su Lan of Clinical Research Centre Hospital Queen Elizabeth, Kota Kinabalu for their help and guidance in statistics and analysis and $\mathrm{Mr}$ (Dr.) Arshad Ali for his contribution towards my thesis.

Heartful thanks also expressed to fellow doctor colleagues, especially Dr. Vidya Pillai and Dr. Nishanti Apporrow and nursing staff in the department of neurosurgery in Queen Elizabeth Hospital for their support and help in data collection.

I also would like to thank everyone whose name were not mentioned here but has aid, guided, and lent hand in the success of this study. Lastly a special thanks to my wife, Dr. Thivashini for her continuous support to me.

\section{References}

1. Baker ML, Hand PJ, Wang JJ, Wong TY : Retinal signs and stroke: revisiting the link between the eye and brain. Stroke 39 : 1371-1379, 2008

2. Edwards MS, Wilson DB, Craven TE, Stafford J, Fried LF, Wong TY, et al. : Associations between retinal microvascular abnormalities and declining renal function in the elderly population: the Cardiovascular Health Study. Am J Kidney Dis 46 : 214-224, 2005

3. Kumar $\mathrm{V}$, Abbas A, Fausto $\mathrm{N}$ : Robbins and Cotran Pathologic Basis of Disease, ed 7. Philadelphia : Elsevier, 2005

4. Liew G, Wong TY, Mitchell P, Cheung N, Wang JJ : Retinopathy predicts coronary heart disease mortality. Heart 95 : 391-394, 2009

5. Mondal RN, Matin MA, Rani M, Hossain ZM, Shaha AC, Singh RB, et al. : Prevalence and risk factors of hypertensive retinopathy in hypertensive patients. J Hypertens (Los Angel) 6 : 241, 2017

6. Ng WK, Goh KJ, George J, Tan CT, Biard A, Donnan GA : A comparative study of stroke subtypes between Asians and Caucasians in two hospital-based stroke registries. Neurol J Southeast Asia 3 : 19-26, 1998

7. Ong YT, Wong TY, Klein R, Klein BE, Mitchell P, Sharrett AR, et al. : Hypertensive retinopathy and risk of stroke. Hypertension 62 : 706-711, 2013

8. Patton N, Aslam T, Macgillivray T, Pattie A, Deary IJ, Dhillon B : Retinal vascular image analysis as a potential screening tool for cerebrovascular disease: a rationale based on homology between cerebral and retinal microvasculatures. J Anat 206 : 319-348, 2005

9. Sabanayagam C, Shankar A, Klein BE, Lee KE, Muntner $P$, Nieto FJ, et al. : Bidirectional association of retinal vessel diameters and estimated GFR decline: the BEaver Dam CKD Study. Am J Kidney Dis 57 : 682-691, 2011

10. Sairenchi T, Iso H, Yamagishi K, Irie F, Okubo Y, Gunji J, et al. : Mild retinopathy is a risk factor for cardiovascular mortality in Japanese with and without hypertension: the Ibaraki Prefectural Health Study. Circulation 124 : 2502-2511, 2011

11. Tso MO, Jampol LM : Pathophysiology of hypertensive retinopathy. Ophthalmology 89 : 1132-1145, 1982

12. Wong T, Mitchell $P$ : The eye in hypertension. Lancet $369: 425-435$, 2007

13. Wong TY, Coresh J, Klein R, Muntner P, Couper DJ, Sharrett AR, et al. : Retinal microvascular abnormalities and renal dysfunction: the atherosclerosis risk in communities study. J Am Soc Nephrol 15 : 2469-2476, 2004 